Submitted to 1997 Particle Accelerator Conference,

Yancouver, B.C., Canada, May 12-16, 1997*

\title{
Global Orbit Feedback Utilizing Analog and Digital Technologies
}

\author{
O. Singh, Y. Tang, S. Ramamoorthy, S. Krinsky, L.H. Yu \\ NSLS, Brookhaven National Laboratory.
}

\section{Abstract}

At the NSLS, an analog global orbit feedback system is used in regular operations, and a digital global orbit feedback system is available in machine physics studies on the $\mathrm{X}$-Ray Ring. Here, we discuss the relative merits of utilizing analog and digital technology in orbit feedback. Results of experiments are reported characterizing the performance of our analog and digital systems when operated individually or together. We give our thoughts on plans for future development of the orbit feedback systems at the NSLS.

\section{INTRODUCTION}

The analog global orbit feedback systems have been operational on the NSLS storage rings since 1991. These are based on correcting only a few harmonics near the tune [1] and they utilize only one-third of the available pues and trims. The analog systems provide an order of magnitude reduction in orbit motion using inexpensive hardware. The analog feedback controller is wideband and provides a stable feedback system with orbit correction bandwidth of $200 \mathrm{hz}[2,3]$. From dc to $2 \mathrm{hz}$, the harmonic content of the orbit is corrected by a factor of 100 , but at higher frequencies, the correction reduces linearly with frequency reaching to unity at $200 \mathrm{hz}$. We are also developing a digital orbit feedback system $[4,5]$ which has been used during machine physics studies in the $\mathrm{X}$-ray storage ring. It employes up to 25 eigenvectors and all available pues and trims. The digital feedback system provides a significant improvement in slow orbit variation (less than $1 \mathrm{~Hz}$ ), however, its correction bandwidth of $20 \mathrm{hz}$ provides only moderate improvement for fast orbit motion.

We have three basic modes of operating the orbit feedback systems: (1) Run only the analog orbit feedback systerm as is the case during regular operation. (2) Run only digital feedback system, presently only in machine studies. In this case, the digital feedback system uses up to 25 eigenvectors and is highly effective in correcting orbit drift. The eigen values, with no feedback, are given in Table 1. (3) First the analog feedback is turned on, and then the digital feedback is turned on. In this mode, the digital feedback system uses a modified response matrix obtained with the analog system on. Using about 10 eigenvectors, the combined system provides additional orbit correction beyond that provided by the analog system alone. The eigen values, with analog feedback on, are given in Table 2. Fig. 1 and Fig. 2 provide improvement results for horizontal orbit motion in the slow drift and in fast orbit oscillation, respectively. The fast orbit oscillations are due to the NSLS booster, cycling at about $0.7 \mathrm{~Hz}$.

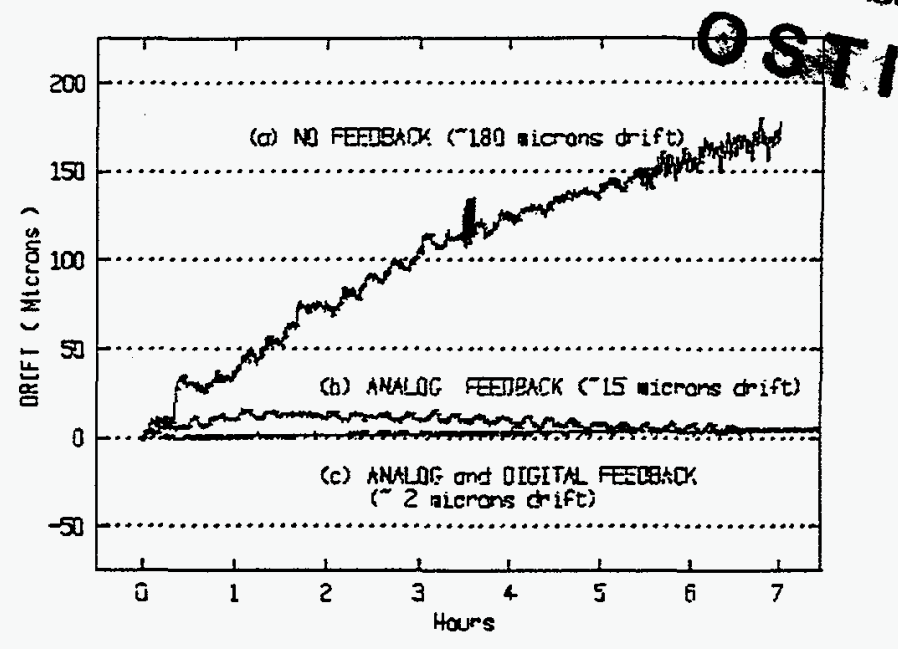

Figure 1: Horizontal orbit slow drift with (a) No feedback, (b) Analog feedback only and (c) Analog and Digital feedbacks.

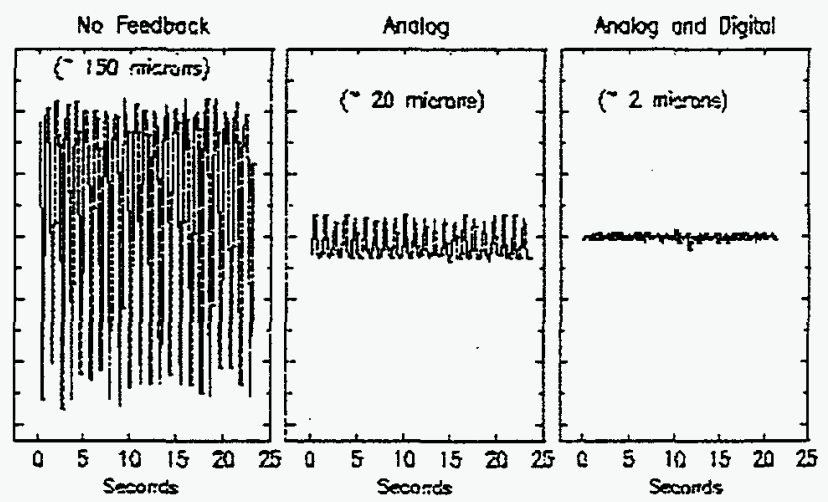

Figure 2: Horizontal orbit fast motion due to cycling NSLS Booster with (a) No feedback, (b) Analog feedback, and (c) Analog and Digital feedbacks.

We have observed that the vacuum chamber moves when it is heated by synchrotron radiation [3]. In the horizontal plane, the amount of this chamber motion is large at some pue locations, resulting in significant orbit measurement errors. This motion must be taken into account in order to use the digital system to improve the horizontal orbit. During studies, when the digital feedback is on, we observe that the photon beam monitored at the diagnostic beamline $\mathrm{X} 28$ shows a larger horizontal beam excursion with digital feedback on, although motion appears to be smaller on the PUEs (see Fig. 1). We are in process of studying the chamber motion and plan to incorporate a correction for PUE motion in order to implement the digital feedback system effectively. 


\section{ANALOG VS DIGITAL}

The set up of our analog and digital orbit feedback systems is shown in Fig. 3. The plant $P$ represents the trim to pue response, the controller $C_{a}$ is the analog feedback element and the controller $C_{d}$ is the digital feedback element. The digital feedback controller $C_{d}$ has two configurations, one without analog feedback and other with analog feedback.

If the background orbit motion is represented by $\mathrm{N}$ as shown in Fig. 3, with both analog and digital feedbacks off, the orbit is given by the uncorrected background orbit motion $\mathrm{N}$. With feedbacks on, it can be easily shown that the residual orbit is $\left[N /\left(1+P\left(C_{a}+C_{d}\right)\right)\right]$ and the performance of a feedback depends on the value of $\left(1+P\left(C_{a}+C_{d}\right)\right)$. At dc, this expression has a value of 100 and the noise reduction will be by two order of magnitude.

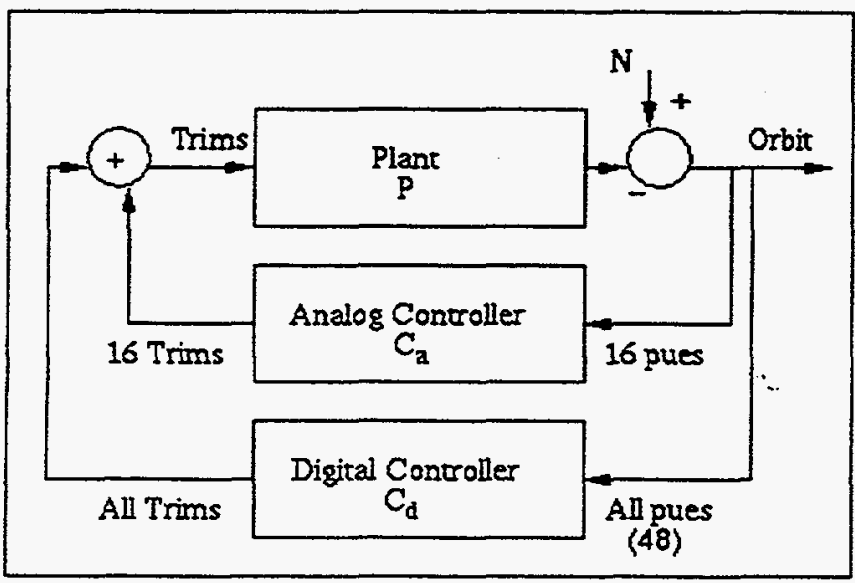

Figure 3: NSLS X-ray ring orbit feedback systems.

Let us comment upon some of the relative merits of analog and digital feedback, based upon our experience, which is constrained by existing hardware. The Bittner-Biscardi receivers provide a very precise, low noise analog output, with a bandwidth of larger than $2 \mathrm{khz}$. The analog feedback systems we have built utilizing the output of the receivers achieve high resolution and wide bandwidth in a straightforward and economical manner. For our relatively small storage rings, utilizing 16 (X-ray ring) or 8 (VUV ring) pues and trims is easy, and provides for a significant improvement in orbit stability. One downside of the analog system is the restriced flexibility in the choice of pues and trims, which limits studies of the dependence of system performance on the design choices of pues. trims and filters. For large rings, requiring more pues and trims, the analog approach may become less attractive.

In our implementation of a digital system, the achievement of submicron resolution, low noise, and high bandwidth has been more difficult than in the analog system. The noise level in the analog to digital converter (ADC) hardware results in a measurement error of a few microns. The bandwidth of the digital controller is presently limited in our case due to ADC sampling limit of $550 \mathrm{~Hz}$, and the speed of the cpu processor used to compute and implement the orbit correction. However, these difficulties may be less restricting when designing a new system, and also will be eased by the rapid progress being made in digital components. On the other hand. the great flexibility made possible by the digital approach has already provided us with important benefits. We can easily add or remove pues or trims from the feedback. to assess their effect. Also, we can easily choose from different orbit correction algorithms, and vary the number of eigenvectors included in the correction. In this way, we have achieved new insight into the optimum conditions for feedback. which has even allowed us to improve the analog vertical feedback on the $\mathrm{X}$-ray ring (see Fig. 4). In studies of digital feedback it was noticed that there are 8 vertical eigenvalues of magnitude greater than unity (see table 1), while existing analog feedback was only using 8 PUES and 6 eigenvectors. Based on this observation, we modified the analog vertical orbit feedback to include 16 PUES and 8 eigenvectors, improving the orbit correction by about a factor of 3 . The improvement results are shown in Fig. 4.

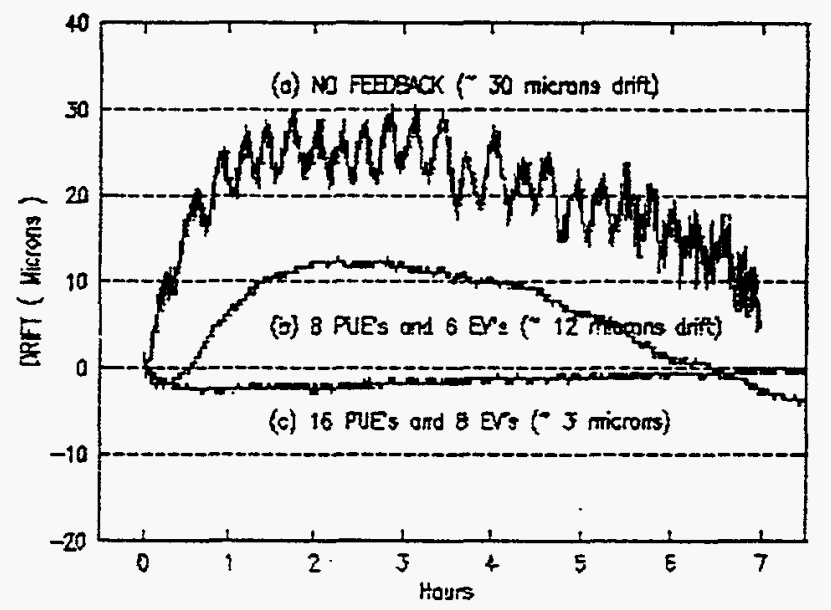

Figure 4: X-Ray vertical orbit drift with (a) No feedback. (b) Analog feedback with 8 PUEs and 6 eigenvectors, and (c) Analog feedback with 16 PUEs and 8 eigenvectors.

\section{FUTURE PLANS}

In short term, we plan to study the performance of digital feedback system by upgrading it with a) new ADC orbit measurement system which will have lower noise level by an order of magnitude, and will sample at a higher rate of about $1 \mathrm{Khz}$; and b) new cpu processor which is faster by a factor of 3 . Simulation of feedback system has shown that these improvements should increase the digital feedback bandwidth from $20 \mathrm{hz}$ to $40 \mathrm{hz}$. We will, also, study the improvement resulting from incorporating correction of pue motion due to vacuum chamber movement.

In long term, we will look into an orbit correction system which utilizes digital feedback only. We expect this system will sample and process orbit data at a rate of several khz. Also, this system should integrate with an orbit measurement system which incorporates correction for PUE's 


\begin{tabular}{|c|r|r||}
\hline \hline $\begin{array}{c}\text { Eigen } \\
\text { number }\end{array}$ & Horizontal & Vertical \\
\hline 01 & 17.62 & 26.88 \\
02 & 11.23 & 16.36 \\
03 & 3.07 & 4.89 \\
04 & 2.97 & 4.65 \\
05 & 2.09 & 3.68 \\
06 & 1.68 & 3.44 \\
07 & 1.64 & 3.01 \\
08 & 1.53 & 2.58 \\
\hline 09 & 1.19 & .30 \\
10 & .98 & .29 \\
11 & .95 & .29 \\
12 & .72 & .28 \\
13 & .70 & .28 \\
14 & .63 & .28 \\
15 & .62 & .28 \\
16 & .45 & .23 \\
17 & .34 & .13 \\
18 & .28 & .13 \\
19 & .27 & .12 \\
20 & .26 & .12 \\
21 & - & - \\
22 & - & - \\
\hline \hline
\end{tabular}

Table 1: Eigenvalues of horizontal and vertical response matrix with no feedback. For vertical, note that there are 8 eigenvalues of magnitude greater than unity.

motion.

\section{ACKNOWLEDGEMENTS}

This work was performed under the auspices of the U.S. Department of energy undercontract DE-AC02$76 \mathrm{CH} 00016$.

\section{REFERENCES}

[1] L.H. Yu, R. Biscardi, J. Bittner, A.M. Fauchet, S. Krinsky, R.J. Nawrocky, J. Rothman, O. Singh and K.M. Yang, " Real Time Orbit Feedback System for NSLS X-ray Ring", Proc. IEEE Particle Accelerator Conf., San Franscisco, Vol 4, p. 2542, 1991.

[2] O. Singh, "Electron beam stabilty and beam peak to peak motion data for NSLS X-ray Storage Ring", Proc. IEEE Particle Accelerator Conf., Washington, D.C.,Vol. 3, p. 2254,1993.

[3] J. Safranek, O. Singh and L. Solomon, "Orbit Stability Improvement at the NSLS X-ray Ring", Proc. IEEE Particle Accelerator Conf.. Dallas, Tx, Vol 4, p. 2711,1995.

[4] A. Friedman and E. Bozoki," A Digital Feedback System for Transverse Orbit Stabilization in the NSLS Rings", NIM A352, p. 393, 1995.

\begin{tabular}{||c|r|r||}
\hline $\begin{array}{c}\text { Eigen } \\
\text { number }\end{array}$ & Horizontal & Vertical \\
\hline 01 & 1.34 & .30 \\
02 & 1.27 & .29 \\
03 & 1.06 & .29 \\
04 & .89 & .29 \\
05 & .74 & .28 \\
06 & .71 & .28 \\
07 & .62 & .28 \\
08 & .59 & .23 \\
09 & .48 & .20 \\
10 & .40 & .14 \\
\hline 11 & .32 & .13 \\
12 & .30 & .13 \\
13 & .28 & .12 \\
14 & .28 & .12 \\
15 & .27 & .12 \\
16 & .24 & .11 \\
17 & .23 & .11 \\
18 & .20 & .11 \\
19 & .05 & .10 \\
20 & .05 & .10 \\
21 & - & - \\
22 & - & - \\
\hline \hline
\end{tabular}

Table 2: Eigenvalues of horizontal and vertical response matrix when analog feedback is ON with 16 pues and 8 eigenvectors. Only 10 eigenvectors are utilized by the digital feedback in this case.

[5] E. Bozoki, A. Friedman, S. Rammamorthy, O. Singh and Y. Tang." Operations with the Digital Orbit Feedback System in the NSLS X-ray Ring", Proc. of the fifth European Particle Accelerator Conf., Sitges (Barcelona), Vol 3, p. 1911, 1996. 


\section{DISCLAIMER}

This report was prepared as an account of work sponsored by an agency of the United States Government. Neither the United States Government nor any agency thereof, nor any of their employees, makes any warranty, express or implied, or assumes any legal liability or responsibility for the accuracy, completeness, or usefulness of any information, apparatus, product, or process disclosed, or represents that its use would not infringe privately owned rights. Reference herein to any specific commercial product, process, or service by trade name, trademark, manufacturer, or otherwise does not necessarily constitute or imply its endorsement, recommendation, or favoring by the United States Governmeat or any agency thereof. The views and opinions of authors expressed herein do not necessarily state or reflect those of the United States Government or any agency thereof. 


\section{DISCLAMMER}

Portions of this document may be illegible in electronic image products. Images are produced from the best available original document. 